\title{
ASPECTOS CONTEXTUALES DEL SURGIMIENTO DE TERAPIA OCUPACIONAL EN LA ARGENTINA
}

\author{
CONTEXTUAL ASPECTS OF THE EMERGENCE OF OCCUPATIONAL THERAPY IN
} ARGENTINA

\section{Julieta Briglia ${ }^{1}$, Agustina García Sartirana ${ }^{2}$.}

\section{Resumen}

El presente trabajo pretende caracterizar algunos aspectos contextuales previos y posteriores a la apertura del primer centro de enseñanza de Terapia Ocupacional en la Argentina, la Escuela Nacional de Terapia Ocupacional en el periodo histórico que va desde 1946 a 1965.

Este artículo fue escrito a partir de nuestra actividad como becarias ${ }^{3}$ en el marco de un proyecto de investigación dirigido por Lic. TO Mariela Nabergoi y conformado por un equipo de docentes investigadores del Instituto de las Ciencias de Rehabilitación y el Movimiento de la Universidad Nacional de San Martín.

Para cumplir con el objetivo propuesto realizamos un análisis de fuentes secundarias que describan el contexto políticoeconómico, social, educativo y sanitario en Argentina durante el periodo estudiado y que traten el tema del surgimiento de Terapia Ocupacional en la Argentina.

Al reflexionar sobre los factores contextuales que permitieron el surgimiento de la TO en el país, podemos afirmar que la epidemia de poliomielitis de 1956 fue, tal como señala buena parte de la bibliografía relevada sobre la historia de TO en nuestro país, una situación desencadenante de la necesidad socio-sanitaria de formar recursos humanos para atender las secuelas de la enfermedad. No obstante, remarcamos la importancia de evitar concebir a la epidemia en términos de causa-efecto con respecto a la creación de la formación profesional en TO, debido a que no puede ser aislada de los contextos político-económico, social, educativo y sanitario.

\section{Palabras clave:}

ENTO - contexto político-sanitario - Terapia Ocupacional - Argentina.

\section{Abstract}

This paper aims to characterize some contextual aspects before and after the opening of the first educational institution for Occupational.

Therapy in Argentina, the National School of Occupational Therapy in the historical period that runs from 1946-1965.

This article was written based on our work as scholars in the framework of a research project led by Mariela Nabergoi and comprised by a team of teacher researchers from the Institute of Rehabilitation Sciences and the Movement of The National University of San Martín.

To meet the proposed objective we performed an analysis of secondary sources which describe the political-economic, social, educational and health contexts in Argentina during the period studied and which address the issue of the emergence of Occupational Therapy in Argentina.

1 Residente de Terapia Ocupacional del Gobierno de la Ciudad de Buenos Aires en Hospital de Emergencias Psiquiátricas T. de Alvear Correo Postal: Maure 4109 Ciudad Autónoma de Buenos Aires. Argentina CP: 1427 Teléfono: $0054-15$ 3001 0207 E-mail:julietabriglia86@gmail.com

2 Residente de Terapia Ocupacional del Gobierno de la Ciudad de Buenos Aires en Hospital de Rehabilitación M. Rocca Correo Postal: Pringles 1286, Ramos Mejía, Buenos Aires. Argentina CP: 1704 Teléfono: 0054- 1530159653 E-mail: toagustinagarcia@gmail.com

3 Beca de entrenamiento de la Comisión de Investigaciones Científicas (CIC) de la Provincia de Buenos Aires 2011. Este trabajo fue realizado en calidad de becarias en el marco del proyecto de investigación "Creación e institucionalización de la Terapia Ocupacional en Argentina. Los comienzos de la formación y el desempeño profesional de terapeutas ocupacionales en Argentina desde la apertura de la Escuela Nacional de Terapia Ocupacional" Directora: Lic. TO Mariela Nabergoi, Co-directora: Lic. TO Marcela Mattei. Instituto de las Ciencias de Rehabilitación y el Movimiento (ICRM) de la Universidad Nacional de San Martín (UNSaM). 
Reflecting on the contextual factors that allowed the emergence of OT in the country, we can state that the polio epidemic of 1956 was, as pointed out in much of the literature on the history of OT relieved in our country, a situation triggering the need for social care to form human resources to attend to the effects of the disease. However, we emphasize the importance of avoiding conceiving the epidemic in terms of cause and effect with respect to the creation of vocational training in OT, because it cannot be isolated from the broader political-economic, social, educational and health contexts.

Key words:

ENTO - Political sanitary context - Occupational Therapy - Argentina.

\section{INTRODUCCIÓN:}

El presente trabajo se inscribe en el marco del proyecto "Creación e institucionalización de la Terapia Ocupacional en Argentina. Los comienzos de la formación y el desempeño profesional de terapeutas ocupacionales en Argentina desde la apertura de la Escuela Nacional de Terapia Ocupacional". El mismo es dirigido por Lic. TO Mariela Nabergoi y conformado por un equipo de docentes investigadores del ICRM UNSaM. Continuando la línea de investigación abierta por el equipo desde el año 2007, el proyecto se propone profundizar la revisión sistemática y relevamiento de documentos y testimonios de la historia de los acontecimientos relevantes ocurridos en el contexto histórico de creación de la Escuela Nacional de Terapia Ocupacional (ENTO) y los primeros años de la formación y desarrollo de la profesión en el país.

En este marco, el plan de trabajo se propone contribuir al cumplimiento del siguiente objetivo: describir los aspectos contextuales previos y posteriores a la apertura del primer centro de enseñanza de Terapia Ocupacional en la Argentina en las dimensiones económica, política, social, educativa y sanitaria.

\section{Metodología}

Como metodología se realizó un rastreo del estado del arte mediante el análisis de fuentes secundarias que describieran los contextos económico, político, social, educativo y sanitario en el periodo histórico que va desde 1946 a 1965 en Argentina y que traten el tema del surgimiento de Terapia Ocupacional en dicho país. Se relevó información bibliográfica en asociaciones nacionales, tesis de grado y post-grado, libros, diarios y publicaciones en revistas del área.

\section{Resultados}

A continuación se desarrollan los resultados parciales del trabajo realizado.

La Terapia Ocupacional surge en la Argentina en el entrecruzamiento de dos etapas bien diferenciadas en relación al contexto político. Anterior a su surgimiento, el país había sido gobernado por el peronismo. Se inició luego una larga etapa de gobiernos de facto alternados con gobiernos democráticos inestables que se extendió hasta 1983. En esta última etapa surge y se desarrolla la carrera de TO en el país.

A través del rastreo del estado del arte pudimos observar que la mayoría de los autores que escriben acerca de los orígenes de TO en la Argentina se refieren a la epidemia de poliomielitis de 1956 como el factor desencadenante de la creación de la carrera (AATO, s/f; Macdonald, 1972; Nabergoi \& Mattei et al., 2001; Ramacciotti, 2006; Risiga \& Monzón, 2003; Warchavski \& Sbriller, 1990). Consideramos importante ampliar la mirada al respecto para poder reflexionar qué otros factores contextuales permitieron que éste sea un hecho desencadenante o si hubo otros que colaboraron en el surgimiento de la TO en el país.

El desarrollo del trabajo se organizará en tres apartados según las siguientes temáticas: aspectos contextuales previos a la epidemia de Poliomielitis de 1956; epidemia de poliomielitis de 1956 y su relación con el surgimiento de TO en Argentina; y aspectos contextuales posteriores a la epidemia de Poliomielitis de 1956.

\section{Aspectos contextuales previos a la epidemia de Poliomielitis de 1956}

El peronismo tuvo su gran etapa de apogeo antes de la epidemia de poliomielitis de 1956 . El primer gobierno de Juan Domingo Perón fue desde 1946 a 1952. En 
1949, Perón reformó la Constitución de 1853, y entre las reformas que realizó se encontraba la posibilidad de reelección. Ello permite que asuma nuevamente desde 1952, que debió llegar a 1958, pero terminó abruptamente en septiembre de 1955 con un golpe de Estado.

Durante el primer gobierno peronista se destaca como un hecho histórico el sufragio femenino, promovido por Eva Duarte unos años antes. En el contexto sanitario, la salud pública apareció como una función importante del Estado, centralizando la gestión. En 1949 se crea el Ministerio de Salud Pública, siendo el primer ministro Ramón Carrillo. Se conformó un sistema único de Seguridad Social, centralizó la administración y la regionalizó mediante las delegaciones sanitarias, creó nuevos hospitales, consultorios externos, servicios intermedios, servicios de apoyo y centros de salud (Perrone \& Teixidó, 2007; Paganizzi, 2007; Svampa, 2010).

En 1955 se produce un golpe de Estado, liderado por la autodenominada "Revolución Libertadora".

Aquí se inaugura la segunda gran etapa-periodo de gobiernos de facto alternados con gobiernos democráticos (Rossi; Ibarra; Ferro s/f.) que empieza en 1955 y se extiende hasta 1983, caracterizada por inestabilidad y conflicto. El conflicto social y la violencia política fueron creciendo en intensidad, incluyendo el fusilamiento con víctimas civiles. Perón y el partido justicialista, aun desde la proscripción, seguirán siendo un factor importante en el panorama político de todo este período.

En 1955 se disuelve el Ministerio de Salud Pública, y se incluyen sus funciones en el Ministerio de Asistencia Social, que a su vez, fue disuelto ese mismo año, al asumir el General Aramburu. De este modo no hubo Ministerio de Salud hasta el año 1958.

Durante este gobierno se anula por Decreto la vigencia de la Constitución de 1949 y se reimplantó la de 1853, prometiendo en su momento convocar a una asamblea constituyente para actualizarla. Como consecuencia en 1957 se agrega el art 14 bis con algunos de los derechos y obligaciones sociales de la Constitución de 1949.

Se produce una reestructuración del sistema sanitario, focalizada en la descentralización, por lo que la salud pasó a ser una responsabilidad exclusiva de las provincias. (Perrone \& Teixidó, 2007).

Dentro del contexto educativo, entre 1955 y 1966 se ubicó la "época de oro" de la universidad argentina. Las autoridades que encararon la "normalización" de la Universidad de Buenos Aires entre 1955 y 1958 se propusieron revitalizar la autonomía y el co-gobierno universitario, la actividad académica y científica de ex- celencia, la creación de nuevas ofertas de formación ${ }^{4}$, la expansión de dedicaciones exclusivas, y la organización de las unidades académicas por departamentos; también se dio una ofensiva política de "desperonización" de la universidad que comprendía la evaluación de la "honradez" y "moralidad" de los candidatos a concurso, esto es, que les imponía la obligación de demostrar públicamente que no disponían de antecedentes comprometidos con el régimen peronista (Buchbinder, 2005). Durante este periodo creció el número de estudiantes y también la inclusión de la mujer en la universidad $^{5}$ (Bonder, 1994).

Por otra parte, en 1955 se firma el Decreto № 6.403, cuyo artículo $N^{\circ} 28$ concede autorización para el funcionamiento de universidades privadas y les permitía expedir diplomas y títulos habilitantes (Luna, 1999; Portal oficial del Gobierno de la República Argentina, 2011).

\section{Epidemia de Poliomielitis de 1956 y su relación con el surgimiento de TO en Argentina}

\subsection{Antecedentes: epidemias anteriores y la creación de ALPI}

La Argentina sufrió reiterados y acentuados brotes de parálisis infantil desde 1906. Entre 1906 y 1932 se produjeron 2.680 casos y en el transcurso de 1932 y 1942 hubo 2.425 enfermos, cifra que se incrementó durante 1942 y 1943, llegando a 2.280 casos (Ramacciotti, 2006).

\footnotetext{
Sociología: se crea la primera carrera en 1957 en la UBA. Psicología: La primera carrera se crea en 1956 en Rosario, Santa Fe. En 1957 se abre en la UBA, en 1958 Córdoba, La Plata y San Luis, en 1959 en Tucumán y en 1966 en Mar del Plata. Trabajo Social: se crea en 1959 con el surgimiento del Instituto de Servicio Social en Buenos Aires. Ciencias de la Educación: en 1953, se crea en Córdoba la carrera de Pedagogía. Ésta daría lugar, a los pocos meses, a la fundación de la Escuela de Ciencias de la Educación y al cambio de denominación de su oferta de grado, llamándose entonces Ciencias de la Educación. Fuente: http://www.ffyh.unc edu.ar/carreras-de-grado/escuela-de-ciencias-de-la-educacion.

5 Cantidad de alumnos en el nivel superior por habitante. Alumnos/10.000 habitantes: 7 en 1914; 32,4 en 1950: 106,7 en 1970. Datos básicos sobre la Educación Superior. Aportes para discursos, debates y propuestas. Área de Articulación de la Educación Superior. Secretaría de Políticas Universitarias. Mónica Marquina; Cecilia Straw. Junio de 2002.
} 
En 1943, se crea ALPI (Asociación para la Lucha contra la Parálisis Infantil) en Buenos Aires, que se constituye como el único centro privado que atendía en forma gratuita a pacientes con discapacidades motoras y con secuelas de Poliomielitis, manteniéndose gracias a la ayuda de la comunidad. ${ }^{6}$

En 1952 la epidemia afectó a 2.579 personas (Ramacciotti, 2006), siendo la cifra más alta de los últimos 20 años, en comparación con las epidemias de las décadas del ' 30 y' 40.

En una conferencia de prensa llevada a cabo el 2 de abril de 1953, el ministro de Salud Pública Ramón Carrillo expresó que los casos de poliomielitis registrados no constituían una epidemia, ya que no se había registrado un caso cada diez mil habitantes. El organismo sanitario era consciente del incremento en el número de casos, pero justificó su aparición diciendo que la situación formaba parte de una "ola epidémica mundial" y que los índices en la Argentina eran menores que los aparecidos en Estados Unidos. De esta manera, el Estado le restaba importancia a esta enfermedad negando explícitamente su carácter de epidemia, e intentaba demostrar su buena situación sanitaria comparándose con Estados Unidos (Ramacciotti, 2006).

Con el correr de los años, se crearon sedes de ALPI en otras provincias de la Argentina, y, a partir de 1955, con la aparición de la vacuna de Salk, se organizaron campañas masivas de vacunación gratuita.

El impacto que la epidemia provocó sobre la sociedad, tenía que ver con que la gran mayoría de los afectados fueron niños que se encontraban desde los recién nacidos hasta los cuatro años (Luna, 1992). No había una cantidad importante de muertes, aunque sí las secuelas en el sistema neuromotor eran visibles y provocaban incapacidades físicas que afectaban una futura inserción en los ámbitos de la vida cotidiana (Testa, 2011; Ramacciotti, 2006).

Otro tópico importante a considerar en este apartado es la influencia europea y norteamericana sobre la historia de América Latina. En Argentina particularmente, esta influencia se puede observar en muchas esferas, sin excluir al campo de la salud. Esto denota que no es circunstancial el interés por la búsqueda de profesionales y modelos teórico-metodológicos en EEUU y Europa durante la epidemia de polio de 1956 para hacer frente a la misma. En este período en particular, ello se expresó en la búsqueda de nuevos modelos de atención desarro-

6 http://www.alpi.org.ar/html/esp/historia.html
Ilados en la posguerra, la solicitud de asesoramiento en materia sanitaria a la Oficina Sanitaria Panamericana, y a través de viajes de expertos promovidos por la Comisión Nacional de Rehabilitación del Lisiado.

\subsection{Epidemia de poliomielitis de 1956 y la creación de la Comisión Nacional de Rehabilitación del Lisiado}

El brote de 1956 toma gran visibilización a través de los medios de comunicación. Se registraron más de $3.000 \operatorname{casos}^{7}$ en 1956, con un promedio de 60 diarios y un $10 \%$ de fallecimientos. Félix Luna (1992) aclara que no todos afligían a los niños y que hubo muchos enfermos con secuelas irreversibles, hasta que hacia el mes de abril de 1956 el brote declina hasta su extinción. Este último dato no concuerda con otros (Testa, 2011) que refieren que en mayo de ese mismo año la enfermedad continuaba en pleno brote.

Daniela Testa (2011) expresa que durante la epidemia de poliomielitis de principios de 1956, se registraron a nivel nacional, aproximadamente 6.500 casos $^{8}$. Esta autora explica que a pesar de una insuficiencia de recursos y de infraestructura sanitaria, y de la inexistencia de planificación por parte del Estado frente a los cíclicos brotes de esta enfermedad presente en Argentina, el gobierno de Aramburu aprovechó dos hechos que aumentaron las posibilidades de controlar la enfermedad en un mediano plazo, y permitían una visibilización a través de los medios de comunicación del problema $^{9}$ : la eficacia comprobada de la vacuna Salk y

La suma los casos de poliomielitis relevados por la Dirección de la Lucha contra las Epidemias y Endemias del Ministerio de Asistencia Social y Salud Pública desde julio de 1955 hasta febrero de 1956 en el país, a pesar de que algunos números del diario no se leen nítidamente, sería menor a 1000. Fuente: "Siguen Combatiendo los Nuevos Casos de Parálisis", Diario Clarín, febrero de 1956. Ver Anexo, Noticia $\mathrm{N}^{\circ} 1$.

Esta referencia oficial contrasta ampliamente con los números de casos relevados posteriormente por investigaciones y artículos citados en este artículo.

8 Ídem.

9 Ver en Anexo las noticias ํo2 "Se vacunará con gamma-globulina a 300.000 niños". Diario La Nación, 14 de marzo de 1956 y N³ "Conferenció con el jefe del Estado la Dra. Jessie Writh"s/f., que muestran principalmente la utilización de la epidemia por parte del gobierno de facto para defenestrar al peronismo, y la valorización de los modelos teóricos y el recurso humano extranjeros. 
el reconocimiento de los progresos de la rehabilitación como especialidad médica en ámbitos científicos y sociales internacionales.

Es de destacar que "la primera estructura estatal para dar respuesta al problema de la epidemia instituida en mayo de 1956, que hasta ese momento estaba íntegramente en manos de las organizaciones privadas, fue la Comisión Nacional de Rehabilitación del Lisiado dependiente del Ministerio de Asistencia Social y Salud Pública. Esta institución se creó en lugares físicos muy representativos del peronismo, la Ciudad Infantil y la Ciudad Estudiantil y dio lugar a la implementación de un programa nacional de rehabilitación y a la creación y construcción dentro del mismo predio, de la Escuela de Terapia Ocupacional y la Escuela de Ortesis y Prótesis" (Testa, 2011).

\subsection{La Rehabilitación y el surgimiento de Terapia Ocupacional}

Ya desde 1947 se habían contratado médicos especializados en el extranjero y se había enviado a otros países a médicos jóvenes, enfermeras y kinesiólogas para que perfeccionaran sus conocimientos acerca del tratamiento $y$, a su regreso, pudieran difundirlos (Ramacciotti, 2006).

Desde los primeros avances en la creación de la carrera de Terapia Ocupacional, el enfoque de la rehabilitación fue importado desde EEUU y Europa y se utilizó ese recurso humano para la capacitación inicial:

- 1956: Miss Betty Hollings llega desde Inglaterra para dictar el primer curso de auxiliares de TO.

- 1957: ALPI organiza el primer curso acelerado dictado por Ruby Kroom proveniente de California, EEUU.

- 1958: la Organización Mundial de la Salud y la Fundación "Hermana Elizabeth Kenny" (Minneapolis, EEUU) envían a Helen Dalhstrom y Vivian Hannan en calidad de asesoras durante un año (Fortain, en Monzón y Risiga, 2003; Bottinelli, Nabergoi y otros, 2009).

Consideramos que los factores contextuales descriptos hasta aquí fueron conformando una escena política compleja que permitió, con el fin de combatir las secuelas que dejó la gran epidemia de polio de 1956, crear la Comisión Nacional de Rehabilitación del Lisiado y algunos años más tarde la carrera de TO.
Es en 1959 cuando se inaugura oficialmente la Escuela Nacional de Terapia Ocupacional (ENTO) con terapistas ocupacionales del "Dorset House School of Oxford" -Inglaterra-. La Directora fue la TO Miss Mac Donald quien coordinó la escuela junto con las TO británicas Miss Barbara Allan (vicedirectora), Miss Dorotea A. Ricket (instructora) y Miss Hillary Schlesinger (instructora). Las inglesas permanecieron dirigiendo la formación en la ENTO hasta 1964, cuando asume la dirección la TO argentina Marta Fortain (Sbriller, 1997; Nabergoi, Mattei, y otros, 2011).

Otros factores que no se pueden dejar de considerar para reflexionar sobre el contexto del origen de la TO en la Argentina, son las profundas modificaciones que se dieron en el periodo estudiado en relación al género femenino y la inclusión en la universidad. Si bien el sufragio femenino fue una de las inclusiones más notorias del género en la sociedad de aquella época, en relación al contexto educativo, teniendo en cuenta que en 1951 se graduaba una mujer por cada 4 varones y en 1966 se graduaba una mujer por cada 2 varones (5), podemos decir que en el período analizado se duplicó la cantidad de mujeres graduadas en la universidad. Particularmente, terapia ocupacional es una carrera que desde su génesis fue creada con la participación de mujeres y ofrecida exclusivamente para el género femenino. Esto no fue una excepción en la Argentina.

\section{Aspectos contextuales posteriores a la epidemia de Poliomielitis de 1956}

El 23 de febrero de 1958 la Revolución Libertadora convocó a elecciones con proscripción total del Partido Justicialista. Las mismas fueron ganadas por Arturo Frondizi (de la Unión Cívica Radical Intransigente). Su periodo de gobierno se caracterizó por adoptar el desarrollismo. La política desarrollista permitió un relativo crecimiento de las industrias automotriz, siderúrgica y petroquímica. En relación al contexto político, Frondizi volvió a habilitar el peronismo electoralmente, manteniendo la prohibición a Juan Domingo Perón de presentarse como candidato y volver al país (Pigna, n/d 2012).

En torno al contexto sanitario, debido a que en el período comprendido entre 1955 y 1958 no hubo Ministerio de Salud, el Presidente Arturo Frondizi volvió a crear el organismo bajo el nombre de Ministerio de Asistencia Social y Salud Pública. Héctor V. Noblía y Tiburcio Padilla fueron sus Ministros hasta 1962. 
En materia de medicina preventiva se planificaron y ejecutaron numerosos programas de exámenes de salud e inmunización a segmentos vulnerables del interior del país; se realizaron diversas campañas de vacunación en plazos y niveles de cobertura inéditos (la triple, B.C.G., antipolio, doble y viruela); se declaró la obligatoriedad de la vacuna antituberculosa (Ley 14.837) y se modificó la normativa referida a la notificación de enfermedades transmisibles (Ley 12.317, modif. por Ley 15.465). (Gómez Paz, 2008).

Dentro del ámbito educativo, en agosto de 1958 el gobierno se propuso revalidar el famoso y cuestionado artículo $\mathrm{N}^{\circ} 28$. Hombres muy prestigiosos de la Iglesia y el Ministro de Educación fueron los artífices del proyecto. Los 7 rectores de las universidades nacionales pidieron al presidente que no innovara "para no alterar la vida institucional y académica" (Luna, 1999). Finalmente, se elaboró una propuesta que fue aceptada por todos los sectores: el artículo $N^{\circ} 28$ se modificaría a fin de que los títulos expedidos por las universidades privadas estuvieran sujetos a la habilitación del Estado.

Durante este gobierno se puso el acento en la capacitación de los recursos humanos profesionales y técnicos. Siguiendo las recomendaciones de los consultores del período anterior, se creó la Escuela de Salud Públi$\mathrm{Ca}^{10}$ con programas destinados a la formación de profesionales sanitaristas y de técnicos en radiología, laboratorio, hemoterapia, áreas que efectivamente tenían graves carencias a nivel técnico asistencial (Gómez Paz, 2008; Perrone \& Teixidó, 2007).

Esta preocupación por la formación profesional llevó al Ministro Noblía a la creación de un plausible sistema de residencias médicas hospitalarias que quedó definido como un sistema de educación profesional para graduados en escuelas médicas, con capacitación en servicio, a tiempo completo y en un plazo determinado a fin de prepararlos para la práctica integral científica, técnica y social de una especialidad (Res. 1.788/1960). (Gómez Paz, 2008).

Esta idea de completar y afirmar la capacidad profesional médica fue objeto de debate en la sede de la Asociación Médica Argentina (noviembre de 1959) y al mes siguiente en el ámbito del Ministerio, oportunidades éstas en las que a través de mesas redondas se evaluaron las posibilidades de viabilidad del sistema (Gómez Paz, 2008).

\footnotetext{
10 Res. 1.580 del 3 de julio de 1959.
}

También fue en esta época en el ámbito de la medicina que se dejó de lado el antiguo practicantado introduciéndose la figura del "Practicante-estudiante", becado que tenía que estudiar: Psicología de grupo, Factores Económicos, Patología Social, Caracterología, Psicología Social, Bases de Administración, Educación Sanitaria, Terapéutica Social y Administración Hospitalaria, disciplinas éstas que no figuraban en los programas de estudio de la Facultad de Medicina (Gómez Paz, 2008).

En 1959 se crean la Universidad Tecnológica Nacional (UTN) y el Consejo Nacional de Educación Técnica (CONET). Surgen las Escuelas Nacionales Técnicas (ENET).

En relación al contexto político, el 29 de marzo de 1962 se produce un levantamiento militar liderado por el general Raúl Poggi. El hecho que precipitó este levantamiento fue la victoria del peronismo en las elecciones realizadas el 18 de marzo, en diez de las catorce provincias existentes en aquel momento, incluyendo a la Provincia de Buenos Aires, donde triunfó el dirigente sindical textil Andrés Framini. Frondizi inmediatamente intervino las provincias en las que había ganado el peronismo, pero el golpe fue indetenible. El presidente es detenido por los militares en la Isla Martin García, se negó a renunciar ("no me suicidaré, no renunciaré y no me iré del país"11).

Finalmente, José María Guido, radical intransigente que presidía la Cámara de Diputados, juró como presidente en el palacio de la Corte Suprema de Justicia. Guido, a pesar de ser civil, cumplió con las órdenes militares, anuló las elecciones realizadas el 18 de marzo, clausuró el Congreso, volvió a proscribir al peronismo, intervino todas las provincias y designó un nuevo equipo económico.

Durante este periodo fueron ministros de Asistencia Social y Salud Pública Tiburcio Padilla y Horacio Rodríguez Castells.

Guido convoca a elecciones presidenciales limitadas el 7 de julio de 1963. Resulta electo Arturo Umberto Illia (candidato de la Unión Cívica Radical del Pueblo). En segundo lugar se encontró el voto en blanco que muchos peronistas utilizaron como forma de protesta.

Illia eliminó las restricciones electorales y políticas respecto al peronismo, aunque no sobre Juan Perón. También legalizó al Partido Comunista.

\footnotetext{
11 La frase fue pronunciada por Arturo Frondizi dos días antes del golpe, el 27 de marzo de 1962, y se convirtió en una de las frases célebres de la historia argentina.
} 
En el contexto económico, durante esta década retoman nuevo impulso las teorías de desarrollo y la planificación económica y social, teorías que se extendieron al ámbito de la salud y que se institucionalizaron en el Consejo Nacional de Desarrollo (CONADE) (Perrone \& Teixidó, 2007). Este gobierno tuvo una política de ordenamiento del sector público, de disminución de la deuda pública y de impulso a la industrialización.

Se creó la Sindicatura de Empresas del Estado, para un control más eficaz de las empresas públicas.

Se anularon los contratos de concesión de explotación petrolera, firmados bajo el gobierno de Arturo Frondizi con empresas privadas extranjeras, por considerarlos contrarios a los intereses nacionales y porque se habían asignado especiales beneficios a dichas empresas, trasladando el riesgo empresario a la empresa estatal Yacimientos Petrolíferos Fiscales (YPF) ${ }^{12}$.

Se sancionó la Ley $N^{\circ} 16.459$ del salario mínimo, vital y móvil, que estableció el Consejo del Salario, de integración tripartita con representantes del Gobierno, los empresarios y los sindicatos. El salario real horario creció entre diciembre de 1963 y diciembre de 1964 un $9,6 \%$.

Respecto al contexto sanitario, el Dr. Arturo Oñativia fue convocado para ejercer el cargo de Ministro de Asistencia Social y Salud Pública de la Nación. Impulsó toda una serie de iniciativas fundamentales: el proyecto de ley de Reforma del Sistema Hospitalario Nacional y de Hospitales de la Comunidad, la creación del Servicio Nacional de Agua Potable y las leyes de Medicamentos ( $\mathrm{N}^{\circ} 16.462$ y 16.463 , de 1963), conocidas como "Ley Oñativia", que le daban al medicamento un carácter de "bien social" al servicio de la Salud Pública y de la Sociedad. Establecían una política de precios y de control de medicamentos, congelaba los valores a los vigentes a fines de 1963, fijaban límites para los gastos de propaganda, imponían límites a la posibilidad de realizar pagos al exterior en concepto de regalías y de compra de insumos (Storani, 2004). Las presiones en contra de la sanción de esta Ley, en especial por parte del Ilamado "Club de París", constituye uno de los motivos esgrimidos por los golpistas a favor del derrocamiento de Illia (Veronelli, 1991).

En 1966 se produce un nuevo golpe militar encabezado por Juan Carlos Onganía.

12 Decretos 744/63 y 745/63 firmados a fines de 1963.

\section{DISCUSIÓN:}

Tal como señala Stagnaro (2006), desde el inicio del trabajo consideramos que si bien al querer contextualizar un periodo histórico cada autor reproduce la historia contándola desde su propia subjetividad, el establecimiento de una secuencia cronológica permite, a la vez, identificar los cambios cualitativos a lo largo del tiempo, trascendiendo la mera sucesión de los hechos.

Con respecto a este último aspecto, algunos autores (Stagnaro, 2006; Buchbinder, 2005) destacan que las temporalidades en relación a la constitución de saberes, campos del saber y su institucionalización, no siempre coinciden con las cronologías políticas, económicas y sociales.

Como ejemplo de esto, en nuestro país pudimos ver diferentes situaciones en las que lo cualitativo de los hechos no se refleja en la cronología, con acontecimientos que surgían algunos como modos de resolución instituyentes por parte de la sociedad, otros como instrumentación de soluciones desde el ámbito político:

Dentro de la llamada "época de oro" de la universidad argentina entre 1955 y 1966 se crean formalmente carreras sociales como psicología, sociología, trabajo social, ciencias de la educación y terapia ocupacional, en una época donde el régimen político estuvo principalmente a cargo de gobiernos de facto y presidentes elegidos democráticamente con proscripciones (Frondizi, 1958-1962 e Illia, 1963-1966). Es decir, que si bien los gobiernos de facto han encarado la política social de un modo bien diferenciado al régimen peronista, de todas formas surgen y perduran en el tiempo importantes cambios que se reflejan en el contexto educativo.

En 1952 la epidemia afectó a 2.579 personas (Ramacciotti, 2006), siendo la cifra más alta de los últimos 20 años, en comparación con las epidemias de las décadas del '30 y '40. Resulta llamativa la negación de la categorización de esta enfermedad como una epidemia en ese entonces por parte del ministro de Salud Pública Ramón Carrillo, quien no ocultaba el número de casos, pero minimizaba de algún modo el problema.

Por el contrario, la situación del brote de 1956 fue utilizada como instrumento político por el gobierno de facto de la "Revolución Libertadora" para culpar al gobierno anterior (Testa, 2011) por la pobre situación económica y sanitaria. Es así como se incluye en la agenda política y es protagonista en los medios de comunicación.

Consideramos que los factores contextuales descriptos hasta aquí fueron conformando una escena política 
compleja que permitió, con el fin de combatir las secuelas que dejó la gran epidemia de polio de 1956, la creación de la Comisión Nacional de Rehabilitación del Lisiado. Este organismo implicó un paso muy importante para la creación de un plan nacional de rehabilitación. Algunos años más tarde en su predio, se Ilevó a cabo la construcción de la Escuela Nacional de Terapia Ocupacional.

Retomando lo planteado en la introducción en relación a la influencia europea y norteamericana sobre la historia de América Latina, pudimos ver también cómo se dio la búsqueda de recursos humanos y modelos teóricos estadounidenses e ingleses para dar inicio a la formación de profesionales argentinos y latinoamerica$\operatorname{nos}^{13}$ en el área de TO. Esto se entrelaza con la cuestión de género, ya que como hemos observado, la mayoría de las personas ligadas a la formación del área de TO que viajaban desde Argentina o Latinoamérica para formarse en el exterior, o las que venían a Argentina desde EEUU e Inglaterra eran mujeres ${ }^{14}$.

Para concluir al reflexionar sobre los factores contextuales que permitieron el surgimiento de la TO en el país, podemos afirmar que la epidemia de poliomielitis de 1956 fue, tal como señala buena parte de la bibliografía relevada sobre la historia de TO en nuestro país, una situación desencadenante de la necesidad sociosanitaria de formar recursos humanos para atender las secuelas de la enfermedad. No obstante, remarcamos la importancia de evitar concebir a la epidemia en tér-

13 Cabe destacar que hubo personas que viajaron desde Latinoamérica para estudiar Terapia Ocupacional en la ENTO. Por lo general fueron becadas desde sus respectivos países, tales como Perú, Chile y Uruguay. Fuente: "Entramados internacionales en la historia de la formación de terapeutas ocupacionales. Indicios en los comienzos de la formación profesional en Argentina". Autores: Nabergoi, M.; Mattei, M.; Zorzoli, F. J. M.; Del Pezzo Bredereke M.; Spallato, N. M.; Bottinelli, M. M.; Muholland, M. J. Trabajo presentado en el XII Congreso Brasilero de Terapia Ocupacional y IX Congreso Latinoamericano de Terapia Ocupacional, octubre de 2011, Sao Paulo, Brasil.

14 La carrera de TO surge en el mundo en el contexto de la primera y segunda guerras mundiales. Desde su origen, debido a que los hombres estaban al frente en la guerra y la mayoría de los que volvían requerían rehabilitación, fueron en su mayoría mujeres quienes se formaron en esta ciencia para rehabilitarlos (Paganizzi, 2007). Además, la profesión trata problemáticas de la vida cotidiana, muchas de ellas pertenecientes al mundo privado, lugar en que la mujer permanecía -excluida del mundo públicollevando a cabo sus roles de ama de casa y cuidadora. minos de causa-efecto con respecto a la creación de la formación profesional en TO, debido a que no puede ser aislada de los contextos político-económico, social, educativo y sanitario.

\section{AgRADECIMIENTOS:}

Agradecemos al equipo de"Creación e institucionalización de la Terapia Ocupacional en Argentina. Los comienzos de la formación y el desempeño profesional de terapeutas ocupacionales en Argentina desde la apertura de la Escuela Nacional de Terapia Ocupacional". A su directora Lic. TO Mariela Nabergoi y Co-directora Lic. TO Marcela Mattei, a los Lic. TO Maureen Muholland, Magdalena Del Pezzo Bredereke, Marcela Bottinelli, Natalia Spallato y Juan Manuel Zorzoli. Agradecemos también al Instituto de las Ciencias de Rehabilitación y el Movimiento (ICRM) de la Universidad Nacional de San Martín (UNSaM) y a la Comisión de Investigaciones Científicas de la Provincia de Buenos Aires.

\section{REFERENCIAS BIBLIOGRÁFICAS:}

Alvarez Guerrero, O. (22 de noviembre de 2011). Arturo Illia. La ortodoxia republicana. Discurso del presidente Arturo Illia del $1^{\circ}$ de mayo de 1965 ante la Asamblea Legislativa. CECIES.

Armus, D. (2010). ¿Qué historia de la salud y la enfermedad? Salud Colectiva, 6 (1), 5-10.

Armus, D. (2002). La enfermedad en la histografía de América Latina Moderna. Asclepio, LIV (2), 41-60.

Asociación Argentina de Terapistas Ocupacionales. (s.f.). http:// www.terapia-ocupacional.org.ar/aat_his.asp. Recuperado el 1 de noviembre de 2011.

Bonder, G. (1994). Mujer y Educación en América Latina: hacia la igualdad de oportunidades. Revista Iberoamericana de Educación (6).

Buchbinder, P. (2005). Historia de las Universidades Argentinas. Buenos Aires: Sudamericana.

Frondizi, A. (1999). Colección Grandes Protagonistas de la Historia Argentina. Planeta.

Garcerón de Gómez, M. S. (2000). Buscando otra mirada de la historia de Terapia Ocupacional. Materia Prima, 4 (15).

Gómez Paz, J.B. (2008). La política de salud en el desarrollismo. Revista de la Asociación Médica Argentina, 4 (121).

Luna, F. (1992). Acindar. 50 años en el país y en el mundo. Edición Institucional. 
Luna, F. (1999). Colección grandes protagonistas de la historia argentina. En F. Luna, Arturo Frondizi. Editorial Planeta.

Mac Donald, E. (1972). Terapéutica Ocupacional en Rehabilitación. Barcelona: Salvay Editores.

Marquina, M. \& Straw, C. (junio de 2002). Datos básicos sobre la Educación Superior. Aportes para discursos, debates y propuestas.

Ministerio de Salud, G. d. (2010). Programa de Residencia de Terapia Ocupacional. Dirección de Capacitación e Investigación.

Nabergoi, M. \& Mattei, M. et al. (octubre de 2011). Entramados internacionales en la historia de la formación de terapeutas ocupacionales. Indicios en los comienzos de la formación profesional en Argentina. Trabajo presentado en el XII Congreso Brasilero de Terapia Ocupacional y IX Congreso Latinoamericano de Terapia Ocupacional. Sao Pablo, Brasil.

Nosiglia, J. E. (1983). El desarrollismo. Centro Editor América Latina.

Paganizzi, L. (2007). Trabajo profesional de terapistas ocupacionales en la Ciudad de Buenos Aires 1964-1996. En L. Paganizzi, Terapia ocupacional psicosocial. Polemos.

Página Web de la Facultad de Filosofía y Humanidades Universidad Nacional de Córdoba, Argentina. Recuperado el 10 de marzo de 2012. http://www.ffyh.unc.edu.ar/carreras-degrado/escuela-de-ciencias-de-la-educacion

Perrone, N., \& Teixidó, L. (2007). Estado y Salud en la Argentina. Revista de Maestría en Salud Pública, 5 (10), 1-46.

Pigna, F. (n/d 2012). Biografía Arturo Frondizi (1908 - 1995). Recuperado el 1 de marzo de 2012 en http://www.elhistoriador. com.ar/biografias/f/frondizi.php

Portal oficial del Gobierno de la República Argentina (s.f.). Recuperado en octubre de 2010, de www.argentina.gov.ar

Ramacciotti, K. I. (2006). Recuperado el 8 de marzo de 2012, de www.slideshare.net/niedfeld/poltica-y-enfermedades-enbuenos-aires-1946-1953

Real Academia Española (2012). (Vigesimo segunda edición).

Revista Materia Prima (1997). Estampas de un tiempo. Materia Prima, 2 (5).

Risiga, M. \& Monzón, A. (2003). Espacio para la reconstrucción de la Memoria. Boletín AATO, 21-25.

Rossi, L.; Ibarra, F.; Ferro C. (s./f.). Historia de la Psicología en la Argentina. Revista de Historia de la Psicología en Argentina 2009, N². Recuperado el 11 de marzo de 2012 en: http:// www.psi.uba.ar/academica/carrerasdegrado/psicologia/informacion_adicional/obligatorias/034_historia_2/Archivos/ inv/revista_virtual_historia_de_la_psicologia2.pdf

Sánchez, A. (2007). Revista Tiroides. Obtenido de www.revistatiroides.com.ar/Revistas/16/RevGlandTir16_01.pdf

Sbriller, L. (1997). Introducción a la terapia ocupacional. Marcos conceptuales. Catálogos.

Spampinato \& Testa, D. (2010). Género, salud mental y terapia ocupacional: algunas reflexiones sobre la influencia de la historia de la historia de las mujeres y la perspectiva de género en nuestras prácticas. Terapia Ocupacional Universidad de Sao Paulo, 21 (2).

Stagnaro, J. C. (2006). Evolución y situación actual de la histografía de la psiquiatría en la Argentina. Frenia, IV 1-37.

Storani, M. E. (diciembre de 2004). Obtenido de www.cancerteam.com.ar/invi023.html

Testa, D. (2011). Poliomielitis: la "herencia maldita" y la esperanza de la rehabilitación. La epidemia de 1956 en la Ciudad de Buenos Aires. Intersitio. Revista Sociológica de Pensamiento Crítico, 5 (2).

Veronelli, J .C.; Veronelli Correch, M. (1991). "Los orígenes institucionales de la Salud Pública en la Argentina". $1^{\text {a }}$ ed. Buenos Aires. OPS/OMS.

Warschavski, G. \& Sbriller, L. (1990). Breve reseña histórica de terapia ocupacional. En G. Warschavski \& L. Sbriller, Terapia Ocupacional. Teoría y Clínica. Ricardo Vergara Ediciones. 